\title{
Concordance between dobutamine Doppler tissue imaging echocardiography and rest reinjection thallium-201 tomography in dysfunctional hypoperfused myocardium
}

\author{
F Larrazet, D Pellerin, D Daou, S Witchitz, C Fournier, A Prigent, C Veyrat
}

\begin{abstract}
Objective-To evaluate the efficiency of the new technique colour Doppler tissue imaging (DTI) by studying the concordance between dobutamine DTI, standard grey scale echocardiography (SE), and rest-reinjection TI-201 tomography (TI) in dysfunctional myocardium.

Patients-23 patients with chronic wall motion abnormalities and proven coronary artery disease $(>70 \%$ diameter stenosis of at least one major coronary artery at angiogram).

Methods-The contractile reserve and the resting perfusion characteristics of dysfunctional myocardial segments were assessed with low dose dobutamine SE and/or DTI (2.5 up to $20 \mathrm{\gamma} / \mathrm{kg} / \mathrm{min})$ and TI on a semiquantitative basis. The DTI or SE data were separately compared with TI, on the basis of a 13 segment ventricular model. The resulting score of combined DTI and SE was also compared with TI. Finally the results obtained from DTI were compared with SE.
\end{abstract}

Results-A total of 142 severely hypokinetic or akinetic segments were visualised. The viability study was feasible in 127 $(89 \%)$ and $121(85 \%)$ segments with DTI and SE, respectively. TI detected viability more frequently than DTI (84 $v$ 61, $\mathrm{p}<0.001)$ and SE $(80 v 50, \mathrm{p}<0.001)$. However, as many viable segments were detected with combined DTI and SE as with TI $(78 v 84$, NS). The $\kappa$ values between TI and SE, DTI or combined SE and DTI were $0.38,0.45$, and 0.57 , respectively, and increased to 0.52 and 0.76 , respectively, for SE and DTI versus TI when mid-anterior and mid-inferior segments only were considered. The $\kappa$ value between SE and DTI was 0.34 .

Conclusions-DTI is a helpful adjunct to SE, when using low dose dobutamine. This combination revealed as many viable segments as TI and showed a better agreement than DTI or SE alone for the assessment of myocardial viable segments evidenced by TI.

(Heart 1999;82:432-437)

Keywords: colour Doppler tissue imaging; hibernating myocardium; thallium 201 single photon emission computed tomography; stress echocardiography
Stress echocardiography is currently performed to detect hibernating myocardium in patients suffering from chronic coronary artery disease. ${ }^{1-5}$ Other techniques such as thallium201 reinjection tomography (TI) and positron emission tomography might offer a good prediction of contractility recovery after myocardial reperfusion or revascularisation. ${ }^{5-8} \mathrm{High}$ concordance between stress echocardiography or TI and positron emission tomography was observed in studies involving small series of patients. ${ }^{89}$ Two previous studies comparing standard echocardiography (SE) and TI paradoxically showed discordances in the specificity for recovery of function. ${ }^{10}{ }^{11}$

Doppler tissue imaging (DTI) was introduced with the hope to give a quantitative echocardiographic approach to myocardial wall velocities and to minimise the subjectivity of SE assessment of ventricular function. ${ }^{12-19} \mathrm{~A}$ visual semiquantitative evaluation of dobutamine on DTI colour images versus grey scale echocardiography has been recently reported, but no reference method was used. ${ }^{20}$

To test the efficiency of this new technique we undertook a study to evaluate the concordance between dobutamine DTI, SE, and TI chosen as a reference method in consecutive patients not selected for their echogenicity suffering from chronic coronary artery disease and dysfunctional myocardium.

\section{Methods}

PATIENTS

Twenty three consecutive patients (three women, mean (SD) age 56 (11) years) with chronic coronary artery disease and resting regional left ventricular dysfunction were included in the study. Informed consent was obtained from all patients. The assessment of both myocardial perfusion and contraction was required in the patients involved in this study for the strategy of revascularisation. TI rather than echocardiography detection improvement in function after revascularisation was chosen as a reference because most interventions were not planned for the patients included in our study. Mean ejection fraction measured by radionuclide ventriculography was 39 (11)\% (range 10-56\%). All patients had a coronary angiogram which exhibited the presence of significant coronary artery stenosis $(>70 \%$ diameter stenosis of at least one major epicardial artery). The study population comprised patients with single $(n=11)$, two vessel $(n=5)$, and three vessel $(n=7)$ coronary
Accepted for publication 13 May 1999 
artery disease. The lesions were located on the left anterior descending coronary artery in 21 patients, the left circumflex coronary artery in seven patients, and the right coronary artery in 13 patients. The exclusion criterion was a recent myocardial infarction (less than three weeks). A previous myocardial infarction, clinical manifestation of cardiac heart failure, and chronic angina pectoris were present in 12, seven, and 12 patients, respectively.

\section{ECHOCARDIOGRAPHIC EQUIPMENT AND}

EXAMINATION

The equipment consisted of a wide angle, phased array two dimensional scanner and a colour Doppler flow and tissue imaging system (Acuson 128 XP 10; Acuson Inc, Mountain View, California, USA). The modifications to obtain only information on tissue have been previously described. ${ }^{12-14}$ The Doppler principle has been validated on rotating tissue models with excellent correlations between Doppler measured velocity and the applied actual speed. Whereas flows, even laminar, have high velocity and weak signal intensity, the reverse occurs for tissues which are phenomena of low velocity and high intensity. Thus, gain amplification used before the quadrature Doppler signal demodulation was reduced in order to avoid the display of intracavitary flows signals, and high pass filters used to reject high intensity tissue Doppler echoes were bypassed with tissue signal directly input into the autocorrelator. Changes in lines and their number of bursts have also been made to increase the frame rate and the sensitivity to low velocities since, as a rule, wall velocities range from $2 \mathrm{~mm} / \mathrm{s}$ up to $20 \mathrm{~cm} / \mathrm{s}$. Temporal resolution of images varies with the frame rate. Velocity maps are the most usual modality. The colour scale reflects the amplitude and direction of velocity. According to the Doppler principle, velocities are positive when approaching toward the transducer (red coded), whereas those away from the transducer are negative (blue coded). The brightest shades correspond to the highest velocities on the colour scale. Velocity scale should be chosen in order to avoid aliasing. The velocity scales usually ranged between 9 and $13 \mathrm{~cm} / \mathrm{s}$. Images were recorded on video, and colour DTI images were digitised online and stored on optical disc for later interpretation. The examination consisted of SE long axis, short axis, and apical four and two chamber views. Once a good quality image had been acquired, the region of interest was imaged by using a regional enhancement sector to increase the frame rate and for optimal colour encoding, with an appropriate filter to avoid blurring of proximal structures such as the chordae tendinae for the posterior wall, and we switched on to DTI.

PROTOCOL FOR DOBUTAMINE ADMINISTRATION After resting images were obtained, dobutamine infusion was started at $5 \mu \mathrm{g} / \mathrm{kg} / \mathrm{min}$ and increased every three minutes to 10,15 , and $20 \mu \mathrm{g} / \mathrm{kg} / \mathrm{min}$. Images were obtained from the standard parasternal and apical views. Continuous monitoring of a 12 lead ECG and blood pressure was performed. Ten patients were on $\beta$ blockers during the diagnostic work up. An increase of more than 10 beats per min from the rest cardiac frequency was considered as the end point of the test.

\section{TI SPECT PROTOCOL}

Stress and four hour rest-reinjection TI-201 single photon emission computed tomography (SPECT) were performed after intravenous administration of $111 \mathrm{MBq}(3 \mathrm{mCi})$ and $56 \mathrm{MBq}(1.5 \mathrm{mCi})$ of the tracer, respectively, with dose schedule to patient's body weight. ${ }^{9}$ TI-201 SPECT was performed within a week of dobutamine echocardiography. Restreinjection TI-201 SPECT acquisition was begun 15 minutes after TI-201 reinjection. All SPECT studies were performed on a one head Gammatome $\gamma$ camera (SOPHA), equipped with a low energy, high resolution collimator. Images were acquired using a circular orbit over $180^{\circ}$, starting at the $45^{\circ}$ right anterior oblique projection and ending at the $45^{\circ}$ left posterior oblique projection, using 32 projections at $35 \mathrm{~s} /$ projection and a pixel size of $6 \mathrm{~mm}$. The energy window was centred on the $70 \mathrm{keV}$ photopeak with a $20 \%$ wide energy window. The uniformity and the centre of rotation of the camera were regularly controlled. Patient or organ motion were corrected when necessary. Prereconstruction filtering of the projection datasets was performed with a Hann filter. Filtered backprojection in conjunction with a ramp filter was used to reconstruct the transaxial tomograms, which were then reoriented into short axis images perpendicular to the long axis of the left ventricle.

SEGMENTATION OF THE LEFT VENTRICLE

A 13 segment ventricular model derived from the 16 segment model was used for the analysis of both isotopic and echographic images. ${ }^{3}$ The segments included the basal, mid, and apical segments of the anterior, septal, lateral, and inferoposterior walls. The apical portion of the left ventricle accounted for the 13th segment.

ECHOCARDIOGRAPHIC ANALYSIS

The images were interpreted by one experienced investigator who was blinded to all other data. The DTI analysis was blinded to the SE interpretation.

SE studies were interpreted by reviewing the videorecording (cineloop) whereas DTI images were usually analysed on early midsystolic frames (fig 1). Frame by frame analysis of the cineloops was used to select the brightest colours corresponding to peak systolic velocity for individual segments. On the one hand the DTI or SE results were separately compared with TI. On the other hand the resulting scores of combined DTI and SE were compared with TI. Data obtained from DTI were compared with SE. Wall motion and velocities were semiquantitated by use of a six grade scoring system. With SE: hyperkinesia $=0$; normal $=1$; mild hypokinesia $=2$; severe hypokinesia $=3 ;$ akinesia $=4 ;$ and dyskinesia $=5$. 


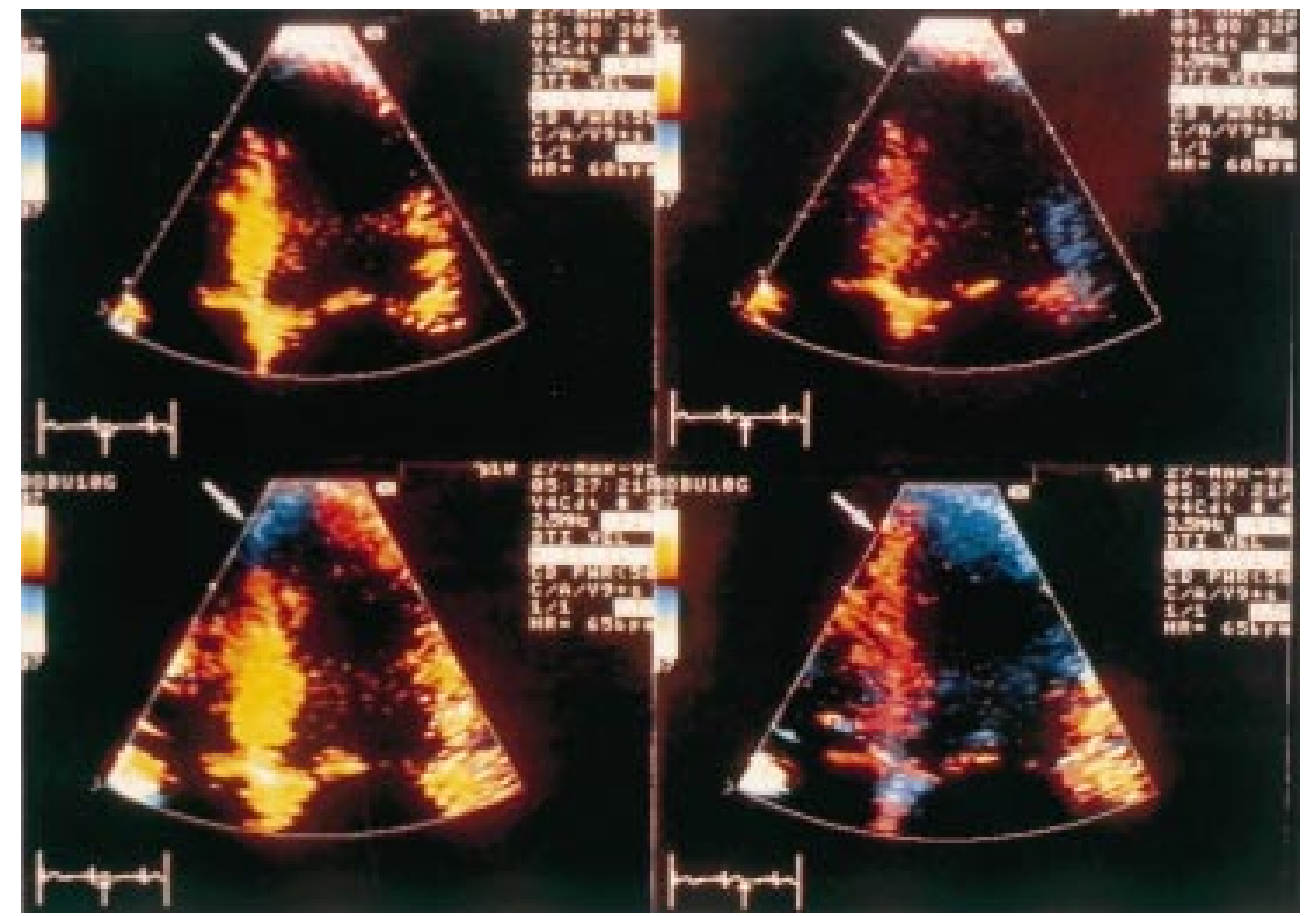

Figure 1 Viability assessment of the septoapical and lateroapical regions of the left ventricle in a patient with a severe left anterior descending coronary artery stenosis. Top: at rest, the septolateroapical area is not encoded (arrows) in early (left) and late (right) systole. Bottom: at the same periods of the cardiac cycle, wall encoding appears after low dose dobutamine.

With DTI: white $=0$; yellow or green (wall motion toward or away from the transducer, respectively) $=1$; orange or turquoise $=2$; red or dark blue $=3$; dark or poorly coloured with red or blue $=4$; paradoxal colouration $=5$. $\mathrm{A}$ change from grade 3,4 , or 5 to grade 2,1 , or 0 was considered positive for the viability evaluation.

TI-201 SPECT ANALYSIS

Rest-reinjection TI-201 SPECT studies were analysed for the interpretation of myocardial viability by an experienced nuclear physician blinded to the clinical, echocardiographic, and angiographic data (fig 2). For this purpose, the TI-201 uptake was scored visually using a 13

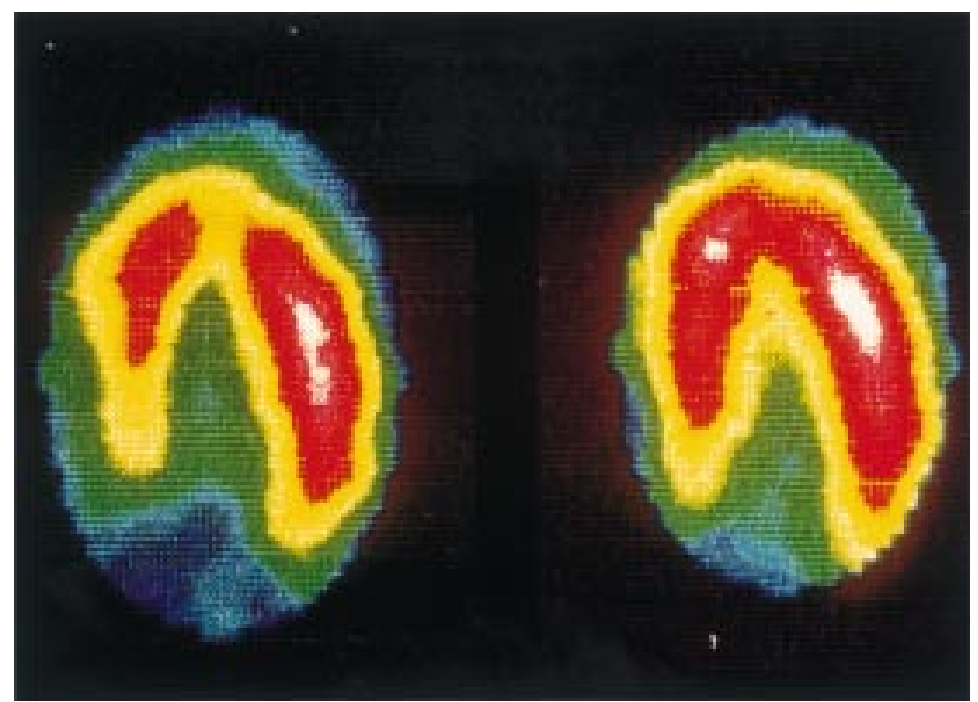

Figure 2 Ischaemia/viability assessment of the septoapical portion of the left ventricle with thallium-201 tomography in the same patient as fig 1. The images show stress on the left and redistribution on the right with the septoapical defect recovery. segment analysis and a five point intensity score for each segment: $0=$ normal; $1=$ mildly reduced; 2 = moderately reduced; 3 = severely reduced; $4=$ absent tracer uptake. ${ }^{21}$

STATISTICAL ANALYSIS

A $\chi^{2}$ test was used to study the relation between echographic and scintigraphic data. Significance was set at $\mathrm{p}<0.05$. Sensitivity, specificity, accuracy, and positive and negative predictive values were obtained from each echocardiographic evaluation with TI-201 SPECT considered as the reference. The agreement between DTI, SE, and TI-201 SPECT was expressed with the $\kappa$ value. Interobserver and intraobserver interpretative variabilities for echocardiography were tested over 116 segments by two observers for the former and within three weeks' time by one observer for the latter. They were expressed by the percent agreement and the $\kappa$ value. As described by Landis and Koch, $\kappa=0-0.2$ suggests slight agreement, $\kappa=0.21-0.40$ suggests fair agreement, $\kappa=0.41-0.60$ suggests moderate agreement, $\kappa=0.61-0.80$ suggests substantial agreement, and $\kappa=0.81-1.0$ suggests almost perfect agreement. ${ }^{22}$

\section{Results}

Of 299 left ventricular segments, 142 (47\%) severely hypokinetic $(\mathrm{n}=87)$ or akinetic $(n=55)$ segments were analysed. Datasets were available in 127 segments (feasibility $89 \%$ ) and 121 segments (feasibility $85 \%$ ) for DTI and SE, respectively. The difficulties encountered to analyse the viability with DTI essentially occurred for the mid-lateral $(\mathrm{n}=4)$ and the anterior $(n=7)$ segments on the apical four and two chamber views. 
Table 1 Operating characteristics and concordance between standard echocardiography (SE), Doppler tissue imaging (DTI), and thallium-201 tomography (TI) in dysfunctional hypoperfused myocardium

\begin{tabular}{lllll}
\hline & & & \multirow{2}{*}{ DTI and } & \\
& SE/TI & DTI/TI & SE/TI & DTI/SE \\
\hline Sensitivity (\%) & 56 & 64 & 81 & 68 \\
Specificity (\%) & 88 & 84 & 77 & 65 \\
Positive predictive value (\%) & 90 & 89 & 87 & 58 \\
Negative predictive value (\%) & 51 & 55 & 67 & 74 \\
Accuracy & 67 & 71 & 80 & 66 \\
$\kappa$ & 0.38 & 0.45 & 0.57 & 0.34 \\
p Value & $<0.0001$ & $<0.0001$ & $<0.0001$ & $<0.0005$ \\
\hline
\end{tabular}

ANALYSIS OF THE CONCORDANCE BETWEEN THE THREE TECHNIQUES

Table 1 shows overall results in term of diagnostic accuracy between SE and TI, between DTI and TI, between combined DTI and SE versus TI, and between DTI and SE. Correspondence in terms of number of segments is detailed in table 2. DTI was at least as accurate as SE for viability detection and seemed more sensitive than SE when TI-201 SPECT was considered as the reference. When DTI and SE were combined, the agreement between echocardiography and TI increased notably. DTI tended to reveal more viable segments than SE but the difference did not reach significance (59 $v 50, \mathrm{NS})$. TI more often revealed viable segments than SE (80 v 50, $\mathrm{p}<0.001)$ or DTI $(84 v 61, \mathrm{p}<0.001)$, but not more than the resulting score of both DTI and SE (84 v 78, NS). When the study concentrated on the mid-anterior and the midinferior segments, the agreement between dobutamine echocardiography and TI was moderate for SE $(\kappa=0.52)$ and substantial for DTI $(\kappa=0.76)$ (table 3$)$.

INTER- AND INTRAOBSERVER VARIABILITIES FOR ECHOCARDIOGRAPHY (116 SEGMENTS)

Intraindividual agreement was $85 \%$ for DTI and SE for the evaluation of the colour of each segment or wall motion analysis, respectively, and $85 \%$ for colour changes induced by dobutamine $(\kappa=0.7)$ versus $79 \%$ for wall motion changes $(\kappa=0.51)$. The interindividual study revealed $89 \%$ agreement for

Table 2 Details for the assessment of viability with SE, DTI, and TI (13 segment ventricular model)

\begin{tabular}{lllr}
\hline \multirow{2}{*}{} & \multicolumn{2}{l}{ Thallium } \\
\cline { 3 - 4 } & & Viable & Not viable \\
\hline \multirow{2}{*}{ SE } & Viable & 45 & 5 \\
\multirow{2}{*}{ DTI } & Not viable & 35 & 36 \\
& Viable & 54 & 7 \\
SE and DTI & Not viable & 30 & 36 \\
& Viable & 68 & 10 \\
& Not viable & 16 & 33 \\
\hline
\end{tabular}

Table 3 Concordance between SE, DTI, and TI in hypoperfused mid-anterior and mid-inferior myocardial segments

\begin{tabular}{lcc}
\hline & $\begin{array}{c}\text { SE/TI } \\
(n=16)\end{array}$ & $\begin{array}{c}\text { DTI/TI } \\
(n=17)\end{array}$ \\
\hline Sensitivity (\%) & 60 & 82 \\
Specificity (\%) & 100 & 100 \\
Positive predictive value (\%) & 100 & 100 \\
Negative predictive value (\%) & 60 & 75 \\
Accuracy & 75 & 94 \\
$\kappa$ & 0.52 & 0.76 \\
\hline
\end{tabular}

colour analysis of each segment with DTI and $52 \%$ agreement for the evaluation of contractility with SE. The percent agreement and the $\kappa$ value for detecting the changes of colour or segmental thickening from baseline to low doses of dobutamine were also better for DTI than for SE. The agreement was substantial for DTI $(86 \%$ agreement, $\kappa=0.74)$ and moderate for SE $(79 \%$ agreement, $\kappa=0.52)$.

\section{Discussion}

The present study shows that an evaluation of TI suspected myocardial hibernation can be more accurately achieved using relatively low doses of dobutamine with the combination of DTI and dobutamine SE than with SE alone. In contrast with DTI or SE alone, the combination of both methods revealed as many viable segments as TI.

DTI has been introduced since 1992 with the hope of quantifying the regional velocity of myocardium. ${ }^{12}$ The quantification of the velocity from either two dimensional or time motion images still necessitates complex time consuming analysis of data from digitised images. Our study shows that a semiquantitative DTI echocardiography analysis can reveal TI suspected hibernating myocardium with an accuracy of $70 \%$ when considered alone and $80 \%$ when the data are associated with SE in patients not selected on the basis of their echogenicity. The $\kappa$ value for the overall analysis ranges from $0.4-0.57$, which represents a moderate agreement between both techniques and notably raised the analysis of the mid-anterior and mid-inferior myocardial segments. ${ }^{22}$

Although earlier investigations comparing dobutamine echocardiography with perfusion scintigraphy ${ }^{23} 24$ and involving a small number of patients showed comparable results, differences in specificity for recovery of function have been more recently reported..$^{11}{ }^{11}$ Hibernating myocardium is defined by its ability to recover function with restoration of adequate perfusion. The evidence of regional recovery of contractility, however, depends on a number of factors including the interobserver and intraobserver interpretative variabilities for the assessment of wall motion (when echocardiography is the imaging technique chosen for later analysis), the technique used for myocardial protection during surgery, the perioperative myocardial infarction in otherwise viable segments, the adequacy of revascularisation, and the speed of recovery ("embalmed myocardium"). ${ }^{25}{ }^{26}$ Recovery of contractility is mainly assessed by standard rest grey scale echocardiography, a method which cannot usually provide evidence of small islands of viable tissue or viable subepicardium in patients with fibrotic subendocardial layers, or viable tissue tethered to scar tissue. ${ }^{26}$

Piérard and colleagues were the first to use low dose dobutamine echocardiography to identify reversible dysfunction after reperfused acute myocardial infarction. ${ }^{8}$ Dobutamine echocardiography has been compared to positron emission tomography in 17 patients. Both techniques were found to be concordant in $79 \%$. Gerber and colleagues studied the ability 
of hibernating myocardium to respond to an inotropic stimulus. ${ }^{27}$ Segments with hibernating myocardium do not improve their function in all cases with dobutamine, especially in patients with severe and globally reduced left ventricular function. These results were confirmed by Pagano and colleagues. ${ }^{28}$ They are probably explained by both the loss of contractility and the limitation of residual coronary flow reserve encountered in hibernation.

Because the results obtained with DTI appear to be closer to TI than the visual assessment of endocardial excursion and myocardial thickening from standard two dimensional images, it raises the hypothesis that colour coded myocardial velocity by DTI not only detects the contraction performance of the myocardium but also its perfusion characteristics (figs 1 and 2). Another interpretation may be that DTI evaluates the transmural wall motion velocities just as TI assesses the transmural myocardial perfusion, whereas SE analysis focuses on the subendocardial layers. In our experience, the tethering effect seems to be minimised by DTI, thus enhancing the detection of suspected viable segments.

A previous visual echocardiographic evaluation of colours had shown encouraging results for the application of DTI to stress echo. ${ }^{20}$ As our data show, a detailed interpretation of DTI images is affected by a lower intra- and interobserver variabilities than SE evaluation of the regional contractility. The interindividual $\kappa$ value was substantial for DTI and moderate for SE. This difference probably reflects the difficulties encountered when distinguishing between mild and severe hypokinesia on SE, whereas the differences between colours-for example, orange and red or turquoise and dark blue-were easier to single out. The poor agreement observed with SE evaluation of wall motion was counterbalanced by a better agreement for the detection of the changes of contractility observed from baseline to low dose dobutamine, thus minimising the risk of discrepancies for the detection of viability.

Our results are consistent with the results of Nagueh and colleagues who observed the same concordance between myocardial contrast echocardiography with intracoronary injection of sonicated albumin and TI $(80 \%, \kappa=0.57)$ as that observed between TI and the combination of DTI and SE. ${ }^{29}$

\section{LIMITATIONS}

In our experience, it remained difficult to analyse the velocities of the anterior and midlateral walls on the four and two chamber views. This limitation may be explained by the suboptimal incidence angle of the ultrasound beam for Doppler velocity encoding. Another limitation might be the choice of restreinjection TI as a reference. Our choice was influenced by a number of factors intervening in function recovery after revascularisation and by the lack of further revascularisation or even incomplete revascularisation proposed to nine of the 23 patients enrolled in the study. Although none of the currently available techniques for the detection of hibernating myocar- dium can be considered superior to the others, specificity to predict functional recovery after revascularisation is probably higher with dobutamine echocardiography than with TI in patients selected for their echogenicity. ${ }^{26}$

Afridi and colleagues showed that patients who recovered rest function after revascularisation had the most improvement in wall motion during dobutamine echocardiography, particularly when a biphasic response was observed. ${ }^{30}$ We did not use the protocol with high doses of dobutamine, particularly in patients with an important left ventricular dysfunction, to avoid the occurrence of a potential threatening ischaemia in normokinetic or moderately hypokinetic adjacent segments and in patients on $\beta$ blockers during the diagnostic work up.

Anatomic misalignment could have also accounted for some of the discrepancy between echocardiographic modalities and scintigraphic study.

Since the study was designed to compare two echocardiography techniques, easily performed, no calculation of the myocardial velocity gradient was made. Accordingly, some dependency of enhanced cardiac translation effect on colour encoded wall velocities cannot be ruled out since translational motion is most likely to be exaggerated when a high dose of dobutamine is given. ${ }^{31}$ In the same way, we estimated wall velocities from the colour shades on the basis of a semiquantitative six grading scale for two reasons. It was similar to the usual grey scale grading on one hand. On the other hand, a different unidirectional colour encoded processing map might enhance the observer's agreement at the expense of a more time consuming procedure. ${ }^{32}$

CONCLUSIONS

Our study suggests that the refinements obtained from DTI increase the ability of echocardiography dobutamine to provide evidence of myocardial hibernating myocardium detected by TI in patients suffering from chronic coronary artery disease. When associated with SE, DTI provided evidence for as many viable segments as TI-201 tomography with a better agreement than dobutamine SE alone. Quantitative studies with either manual or automatic detection of myocardial velocities are needed to evaluate the threshold of velocity variations which would predict functional recovery after revascularisation.

The authors gratefully acknowledge Dr Béatrice Ducot for the statistical analysis and the technical assistance provided by Sabine Chedeville.

1 Cigarroa CG, deFilippi CR, Brickner ME, et al. Dobutamine stress echocardiography identifies hibernating myocardium and predicts recovery of left ventricular function after coronary revascularization. Circulation 1993;88:430-6.

2 La Canna G, Alfiere O, Giubbini R, et al. Echocardiography during infusion of dobutamine for identification of reversble dysfunction in patients with chronic coronary artery disease. 7 Am Coll Cardiol 1994;23:617-26.

3 Afridi I, Kleiman NS, Raisner AE, et al. Dobutamine echocardiography in myocardial hibernation: optimal dose and cardograph in accuracy in predicting recovery of ventricular function after Circulation 1995;91:663-70.

4 Perrone-Filardi P, Pace L, Prastaro M, et al. Dobutamine echocardiography predicts improvement of hypoperfused dysfunctional myocardium after revascularization in patients with coronary artery disease. Circulation 1995;91:
2256-65. 
5 Vanoverschelde J-L, Gerber BL, Pasquet A, et al. Nuclear and echocardiographic imaging for prediction of reversible left ventricular ischemic dysfunction after coronary revascularization: current status and future directions. $\mathcal{F}$ Cardiovasc Pharmacol 1996;28(suppl 1):S27-36.

6 Iskandrian AS, Hakki AH, Kane SA, et al. Rest and redistribution thallium-201 myocardial scintigraphy to predict improvement in left ventricular function after coronary artery bypass grafting. Am f Cardiol 1983;51:1312-16.

7 Ragosta M, Beller GA, Watson DD, et al. Quantitative planar rest-redistribution $201 \mathrm{TI}$ imaging in detection of myonar rest-redistribution $201 \mathrm{TH}$ imaging in detection of myotricular function after coronary bypass surgery in patients tricular function after coronary bypass surgery in patients with severely depress

8 Piérard LA, De Landsheere CM, Berthe C, et al. Identification of viable myocardium by echocardiography during dobutamine infusion in patients with myocardial infarction after thrombolytic therapy: comparison with positon emission tomography. F Am Coll Cardiol 1990;15: 1021-3.

9 Dilsizian V, Perrone-Filardi P, Arrighi JA, et al. Concordance and discordance between stress-redistributionreinjection and rest-redistribution thallium imaging for assessing viable myocardium: comparison with metabolic activity by positon emisson tomography. Circulation 1993 activity by

10 Arnese M, Cornel JH, Salustri A, et al. Prediction of mprovement of regional left ventricular function after surgical revascularization: a comparison of low-dose dobutamine echocardiography with 201TI single-photon emission computed tomography. Circulation 1995;91: 2748-52.

11 Qureshi U, Nagueh SK, Afridi I, et al. Dobutamine echocardiography and quantitative rest-redistribution echocardiography and quantitative rest-redistribution 1997; 95:626-35.

12 McDicken WN, Sutherland GR, Moran CM, et al. Colour Doppler velocity imaging of the myocardium. Ultrasound Med Biol 1992;18:651-4.

13 Sutherland GR, Stewart MJ, Groundstroem WE, et al. Color Doppler myocardial imaging: a new technique for the assessment of myocardial function. F Am Soc Echocardiogr 1994;7:441-58.

14 Miyatake K, Yamagishi M, Tanaka N, et al. New method for evaluating left ventricular wall motion by color-coded tissue Doppler imaging: in vitro and in vivo studies. $\mathcal{7} \mathrm{Am}$ tissue Doppler imaging: in vitiol $1995 ; 25: 717-24$.

15 Uematsu M, Miyatake K, Tanaka N, et al. Myocardial velocity gradient as a new indicator of regional left ventricular contraction: detection by a two-dimensiona tissue Doppler imaging technique. $\mathcal{F}$ Am Coll Cardiol 1995; 26:217-23.

16 Donovan CL, Armstrong WF, Bach DS. Quantitative Doppler tissue imaging of the left ventricular myocardium. Am Heart F 1995;130:100-4.

17 Garcia MJ, Rodriguez L, Ares M, et al. Myocardial wall velocity assessment by pulsed Doppler imaging: characteristic findings in normal subjects. Am Heart $\mathcal{F}$ 1996;132: 648-56.
18 Pellerin D, Cohen L, Larrazet F, et al. Pre-ejectional left ventricular wall motion in normal subjects using Doppler
tissue imaging and correlation with ejection fraction. $A m \mathcal{F}$ tissue imaging and correl

19 Palka P, Lange A, Fleming AD, et al. Differences in myocardial velocity gradient measured throughout the cardiac cycle in patients with hypertrophic cardiomyopathy, athletes and patients with left ventricular hypertrophy due to hypertension. 7 Am Coll Cardiol 1997;30:760-8.

20 Katz WE, Gulati VK, Mahler CM, et al. Quantitative evaluation of the segmental left ventricular response to dobutamine stress by tissue Doppler echocardiography. Am 7 Cardiol 1997; 79:1036-42.

21 Berman DS, Kiat H, Friedman JD, et al. Separate acquisition rest thallium-201/stress technetium-99m sestamibi dual-isotope myocardial perfusion single-photon emission computed tomography: a clinical validation study. 7 Am Coll Cardiol 1993;22:1455-64.

22 Landis JR, Koch GL. The measurement of observer agreement for categorical data. Biometrics 1977;33:671-9.

23 Marzullo P, Parodi O, Reisenhofer B, et al. Value of rest thallium-201/technetium-99m sestamibi scans and dobutamine echocardiography for detecting myocardial viability. Am $\mathcal{F}$ Cardiol 1993;71:166-72.

24 Charney R, Schwinger ME, Chun J, et al. Dobutamine echocardiography and resting-redistribution thallium-201 scintigraphy predicts recovery of hibernating myocardium after coronary revascularization. Am Heart $\mathcal{F}$ 1994;128: $864-9$.

25 Camici PG, Wijns W, Borgers M, et al. Pathophysiological mechanism of chronic reversible left ventricular dysfunc-
tion due to coronary artery disease. Circulation 1997;96: 3205-14

26 Wijns W, Vatner SF, Camici PG. Hibernating myocardium. N Engl F Med 1998;339:173-81.

27 Gerber BL, Vanoverschelde J-L, Bol A, et al. Myocardial blood flow, glucose uptake and recruitment of inotropic reserve in chronic left ventricular ischemic dysfunction: implications for the pathophysiology of chronic myocardial hibernation. Circulation 1996;94:651-9.

28 Pagano D, Bonser RS, Townend JN, et al. Predictive value of dobutamine echocardiography and positon emission tomography in identifying hibernating myocardium in patients with postischaemic heart failure.

29 Nagueh SF, Vaduganathan P, Ali N, et al. Identification of hibernating myocardium: comparative accuracy of myocardial contrast echocardiography, rest-redistribution thallium-201 tomography and dobutamine echocardiography. $\mathcal{A}$ Am Coll Cardiol 1997;29:985-93.

30 Afridi I, Qureshi U, Kopelen HA, et al. Serial changes in response of hibernating myocardium to inotropic stimulation after revascularization: a dobutamine echocardiographic study. F Am Coll Cardiol 1997;30:1233-40.

31 Tsutsui H, Uematsu M, Shimizu H, et al. Comparative usefulness of myocardial velocity gradient in detecting ischemic myocardium by a dobutamine challenge. $\mathrm{f} \mathrm{Am}$ Coll Cardiol 1998;31:89-93.

32 Gorcsan J, Strum DP, Mandarino WA, et al. Quantitative assessment of alterations in regional left ventricular contractility with color-coded tissue Doppler echocardiography: comparison with sonomicrometry an pressure-volume relation. Circulation 1997;95:2423-33. 